\title{
Evaluación de tres métodos de polinización artificial en clones de cacao (Theobroma cacao L.) CCN-51
}

\author{
Evaluation of three methods of artificial pollination in clones \\ of cocoa (Theobroma cacao L.) CCN-51 \\ J. Vera-Chang ${ }^{*}$, R. Cabrera-Verdezoto ${ }^{1 *}$, J. Morán-Morán ${ }^{1}$, K. Neira-Rengifo ${ }^{1}$, \\ R. Haz-Burgos ${ }^{1}$, J. Vera-Barahona ${ }^{1}$, H. Molina-Triviño ${ }^{1}$, O. Moncayo-Carreño $^{1}$, \\ E. Díaz-Ocampo y C. Cabrera-Verdesoto 2
}

\section{RESUMEN}

El objetivo fue evaluar tres métodos de polinización artificial en cacao clonal CCN-51 de siete años de edad, en la Hacienda San Antonio, ubicada en el km 16 de la vía Quevedo-El Empalme. Fueron evaluadas las variables número de flores fecundadas, número frutos sanos cosechados, peso total de fruto $(\mathrm{g})$, número de semillas y rendimiento estimado $\mathrm{kg} \mathrm{ha}^{-1}$. Los tratamientos fueron: T0 = Control polinización natural, T1 = Polinización manual, T2 = Presión de aire con neblinadora sobre las flores del tronco del cacao, T3 = Presión de aire con neblinadora sobre el suelo, el diseño fue completamente al azar con cuatro réplicas. Los datos se analizaron mediante análisis de varianza con la prueba de Tukey ( $\mathrm{p} \geq 0,05$ ) de probabilidad. La variable número de flores polinizadas el T1 polinización manual registró 28 flores, diferente estadísticamente del T0 (Testigo) con 15 flores. Para los frutos sanos cosechados el mejor tratamiento fue el T3 con 6,5 no se encontró significancia estadística. Para el peso total de fruto el polinización natural (testigo) 857,16 g registró el menor valor, diferentes estadísticamente del T2 la presión de aire al tronco y T3 presión de aire al suelo con 938,09 y 937,36 g, respectivamente. Para la variable número de semillas el mejor tratamiento fue le T3 con 43,91 almendras para lo que no se encontró diferencia estadística según Tukey $(\mathrm{p} \geq 0,05)$ y el rendimiento $\left(\mathrm{kg} \mathrm{ha}^{-1}\right)$ el mejor tratamiento fue el T3 con 1.243,64 kg no se encontró significancia estadística. Se concluyó que los mejores métodos para polinizar cacao clonal CCN-51 es aplicando la presión de aire al suelo y tronco.

Palabras clave: cacao, flores, fecundación, frutos, presión de aire, rendimiento.

\begin{abstract}
The objective was to evaluate three methods of artificial pollination in clonal cacao CCN-51 seven-year-old at the Hacienda San Antonio, located at $\mathrm{km} 16$ of the way Quevedo - El Empalme. The evaluated variable were number of fertilized flowers; fruits harvested healthy number, total weight of fruit $(\mathrm{g})$, seed number and estimated yield $\mathrm{kg} \mathrm{ha}^{-1}$. The treatments were: T0 = natural pollination (control), $T 1=$ manual Pollination, $T 2=$ air pressure with neblinadora about flowers trunk cocoa, $T 3=$ air pressure with neblinadora on the floor, the design was completely randomized with four replicates. Data were analyzed by analysis of variance with Tukey test $(p \geq 0.05)$. The variable number of flowers pollinated T1 recorded 28 hand pollination flowers, statistically different from TO (control) with 15 flowers. For healthy fruits harvested the best treatment was 6.5 T3 statistical significance were no found. For total fruit weight natural pollination (control) had the lowest $857.16 \mathrm{~g}$ value $T 2$ statistically different air pressure to the trunk and T3 air pressure to 938.09 and 937.36 soil $g$, respectively. For the variable number of seeds the best treatment was T3 with 43.91 almonds for which no statistical difference according to Tukey $(p \geq 0.05)$ and yield $\left(\mathrm{kg} \mathrm{ha}^{-1}\right)$ was the best treatment with T3 $1243.64 \mathrm{~kg}$ not found statistical significance. It was concluded that the best methods for pollinate clonal cacao CCN-51 is by applying air pressure to the floor and trunk.
\end{abstract}

Key words: cocoa, flowers, fertilization, fruit, air pressure performance.

\footnotetext{
1 Universidad Técnica Estatal de Quevedo (UTEQ), Facultad de Ciencias Pecuarias.Quevedo, Los Ríos, Ecuador

2 Universidad Estatal del Sur de Manabí, Facultad de Ciencias Naturales y de la Agricultura. Manabí, Ecuador.

* $\quad$ Autor por correspondencia: *jverac@uteq.edu.ec, rpcabrera@ute.edu.ec
}

Fecha de Recepción: 8 Septiembre, 2016.

Fecha de Aceptación: 29 Noviembre, 2016.

DOI: $10.4067 /$ S0718-34292016005000033. 


\section{Introducción}

La economía del Ecuador ha estado fuertemente ligada a la producción del cacao (Theobroma cacao $\mathrm{L}$ ). Aproximadamente se exporta alrededor de $110.976 \mathrm{t}_{\mathrm{año}} \mathrm{o}^{-1}$, volumen que representa uno del record histórico de producción y una generación de ingresos cerca de 167 millones de dólares, el $60 \%$ se exporta en grano, el $35 \%$ se destina a la industria para la fabricación de semielaborados y el 5\% se destina a industrias artesanales del país (Enríquez, 2004; Quiroz y Agama, 2005).

Según el Servicio de Información y Censo Agropecuario, SICA (2006), a nivel nacional se cuenta con alrededor de 400.000 ha cultivadas con cacao cuyo rendimiento promedio es de $330 \mathrm{~kg} \mathrm{ha}^{-1} \mathrm{año}^{-1}$, lo que es relativamente bajo para las expectativas del productor resultando antieconómico y no atractivo para ninguna empresa agrícola.

Debido a la baja productividad reflejada en el cacao, hace más de 30 años producto de la selección y cruzamientos controlados con variedades trinitarias, el agrónomo Homero Castro desarrolló el clon $\mathrm{CCN}-51$, caracterizado por ser autocompatible, tolerante a enfermedades y con buena productividad, comparado con el rendimiento tradicional que producen las huertas tradicionales (Crespo y Crespo, 1998).

Se conoce que la planta adulta de cacao bajo condiciones normales puede producir de 6,000 a 10.000 flores por año, de las que solo el $0,01 \%$ llegan a transformarse en frutos. El "cuajamiento o fecundación" puede ser aún más bajo por la falta de insectos polinizadores específicos como la mosca (Forcypomnia spp.); sin embargo, existen otros factores como la edad del material, la incompatibilidad, el exceso de sombreamiento, que influyen directamente en el fructificación (Hardy, 1961; Quiroz et al., 1992; Vera y Cabanilla, 1993).

Investigaciones realizadas en Brasil y Ecuador han demostrado que la presión de aire ocasionada por bombas neblinadoras portátiles, aplicadas sobre cojinetes florales del tallo y al suelo en plantaciones autocompatibles de cacao, pueden incrementar los rendimientos (Urquhart, 1963; Soria, 1977).

Esta investigación tuvo como objetivo determinar el mejor método de polinización que permita incrementar rendimientos del cacao CCN-51 en la zona de Quevedo.

\section{Materiales y Métodos}

La investigación fue realizada en la Hacienda San Antonio, ubicada en el km 16 de la vía QuevedoEl Empalme, cuyas características agroclimáticas son las siguientes: Bosque tropical húmedo, suelo franco, temperatura promedio anual de $24,54{ }^{\circ} \mathrm{C}$, humedad relativa de $86,16 \%$, heliofania 61,05 horas de brillo solar y con una precipitación anual 2.229 $\mathrm{mm}$ promedio. El trabajo fue ejecutado desde marzo hasta octubre de 2009.

\section{Recolección y preparación de flores de cacao}

Como material genético se utilizaron árboles de cacao clonal CCN-51, de siete años de edad en etapa de productiva. Se recolectaron flores frescas de árboles padres en horas de la mañana (9-10 A.M.), estas fueron cuidadosamente colocadas en recipientes plásticos (menos de 3 días de abierta), se identificó la viabilidad del polen por el color blanco aperlado de las anteras (órgano masculino) y el color reluciente de los pétalos. Las flores frescas de los árboles madres se les despejó el pistilo u órgano femenino de la flor donante del polen (padre), se liberó el estambre de la cogulla o concha, con la ayuda de una pinza siguiendo la metodología de Braudeau (1970).

Se utilizaron los siguientes materiales: Pinzas para polinizar de acero inoxidable, tijera de podar, cajones de fermentar de madera $(40$ x $40 \mathrm{~cm})$, machete tipo rabón marca Hansa 15.5, balde plástico 10 litros marca Pika y como equipo una bomba neblinadora marca Cifarelli M1200 EIMA fabricada en Italia año de fabricación 2008, con las siguientes características: $5 \mathrm{Hp}$ (3,6 kw), velocidad de aire $125 \mathrm{~m} \mathrm{segundo}^{-1}$, volumen de aire $20 \mathrm{~m}^{3}$ minuto ${ }^{-1}$, Peso de aire $20 \mathrm{~m}^{3}$ minuto $^{-1}$.

\section{Diseño experimental y análisis estadístico}

Se utilizó un diseño completamente al azar con cuatro tratamientos (Tabla 1) y cuatro repeticiones. Como unidad experimental se utilizaron 20 árboles de cacao clonal por cada tratamiento. Las características del experimento fueron las siguientes: distancia de siembra entre plantas 4 × 3 m, árboles bordes totales 658 , superficie por tratamiento $960 \mathrm{~m}^{2}$, superficie total del experimento $4.000 \mathrm{~m}^{2}$. Para comprobar las diferencias entre tratamientos se empleó la prueba de rangos múltiples de Tukey $(p \geq 0,05)$ se utilizó el programa estadístico SAS (2002). 
Tabla 1. Tratamientos evaluados para analizar los métodos de polinización sobre flores de cacao clonal CCN-51.

\begin{tabular}{cl}
\hline Tratamiento & \multicolumn{1}{c}{ Detalle } \\
\hline T0 & Polinización natural (testigo) flores sin polinizar cuantificadas hasta 1,50 metro en tronco. \\
T1 & Polinización manual artificial suplementaria hasta 1,50 metro en tronco. \\
T2 & Polinización artificial con presión de aire con neblinadora sobre las flores del tronco del cacao superior a \\
& 1,50 metro arriba del suelo. \\
T3 & Polinización artificial con presión de aire con neblinadora sobre el suelo inferior a 1,50 metro del tronco \\
& hacia el suelo. \\
\hline
\end{tabular}

\section{Variables evaluadas:}

Número de flores fecundadas. Se prepararon flores diariamente después de haber realizado las polinizaciones, marcándolas con alfileres de distintos colores con una frecuencia de polinización de cada 7 días, Se polinizó todas las flores disponibles en un rango aproximado de 1 hasta 30 flores por árbol, este procedimiento se lo realiza en ramas principales del árbol hasta una altura de 1,50 metro (Figura 1).

Número de frutos sanos. Los frutos sanos fueron removidos semanalmente en el caso de frutos sanos, fisiológicamente maduros y libres de enfermedades.

Peso total del fruto. Se realizaron los pesajes en gramos de cada uno de los frutos cosechados en cada tratamiento, utilizando una balanza analítica (Figura 1).

Número de semillas. Se realizó en estado de madurez fisiológico y se contaron las almendras por mazorcas.

Rendimiento estimado $(\mathbf{k g})$. Se lo realizó por tratamiento, se registró en la cosecha a los 5 a 6 meses en dependencia de la ubicación del fruto y de la intensidad lumínica y al climaterio, de forma semanal cosechando los frutos maduros provenientes de la polinización. A este se estimó el rendimiento pesando en kilogramos las almendras de las mazorcas cosechadas en cada árbol. Se multiplicó 0,40 , que es el factor de conversión, para obtener su peso seco y, finalmente, fue transformado a rendimiento ( $\left.\mathrm{kg} \mathrm{ha}^{-1} \mathrm{año}^{-1}\right)$.

\section{Resultados y Discusión}

\section{Numero de flores fecundadas, frutos sanos cosechados y peso total de fruto (g)}

Para número de flores fecundadas los mejores tratamientos fueron el T1 y T2 con 28 y 27 flores, diferentes estadísticamente del T0 (testigo) con el menor número de flores fecundadas (15) según Tukey $(\mathrm{p} \geq 0,05)$ (Tabla 2). Este resultado concuerda con lo descrito por Vera y Mogrovejo (1979) quienes atribuyen que el incremento de las flores fecundadas tiene mucha relación con las

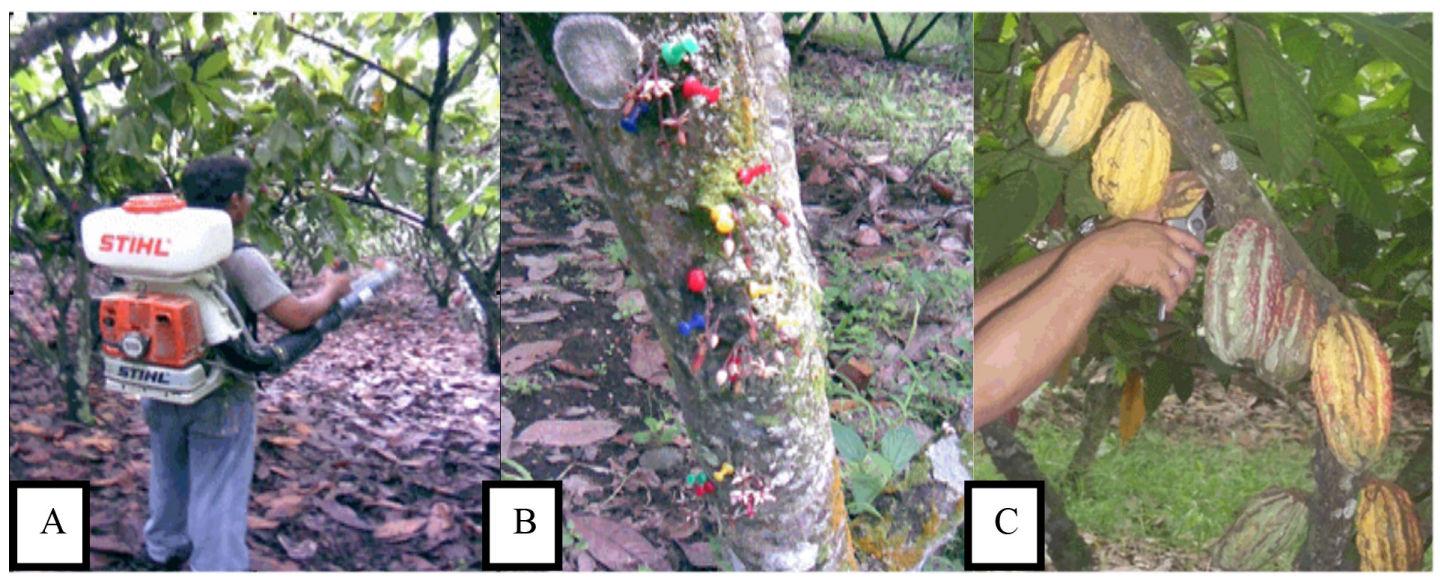

Figura 1. Polinización artificial aplicando presión de aire al tronco y ramas a 1,5 metro del suelo (A). Flores fecundadas polinización manual artificial (7 días) (B). Cosecha de frutos sanos (6 meses) (C). 
Tabla 2. Valores promedios por árbol sobre efecto de los métodos de polinización artificial sobre el número de flores polinizadas, frutos sanos cosechados y el peso total del fruto en cacao clonal CCN-51.

\begin{tabular}{lccc}
\hline \multicolumn{1}{c}{ Tratamientos } & $\begin{array}{c}\text { No flores fecundadas por árbol } \\
(7 \text { días })\end{array}$ & $\begin{array}{c}\text { Frutos sanos cosechados } \\
(6 \text { meses })\end{array}$ & $\begin{array}{c}\text { Peso total de fruto } \\
(\mathrm{g})\end{array}$ \\
\hline 0 Polinización (testigo) & $15 \mathrm{~b}$ & $4,0 \mathrm{a}$ & $857,16 \mathrm{~b}$ \\
1 Polinización manual artificial & $28 \mathrm{a}$ & $5,7 \mathrm{a}$ & $922,96 \mathrm{ab}$ \\
2 P. artificial presión de aire al tronco & $27 \mathrm{a}$ & $6,4 \mathrm{a}$ & $938,09 \mathrm{a}$ \\
3 P. artificial presión de aire al suelo & $22 \mathrm{ab}$ & $6,5 \mathrm{a}$ & $937,36 \mathrm{a}$ \\
Promedio & $\mathbf{2 3}$ & $\mathbf{5 , 7}$ & $\mathbf{9 1 3 , 8 9}$ \\
\hline CV $(\%)$ & $\mathbf{5 , 8 2}$ & $\mathbf{2 6 , 2 4}$ & $\mathbf{4 , 1 5}$ \\
\hline
\end{tabular}

*Medias seguidas por la misma letra no presentan diferencias estadísticamente (Tukey, $\mathrm{p} \geq 0,05$ ).

condiciones climáticas como la humedad, la misma que sobre un $70 \%$, el polen es pegajoso y solo es factible con el traslado de insectos polinizadores (Reyes et al., 1982), y al aplicar la presión de aire tronco permitió liberar e incrementar los granos de polen en la huerta, como también el incremento de los insectos polinizadores. Asimismo, estudios realizados en México con melón, presentaron este comportamiento, esto se respalda con lo reportado Adu-Ampomah et al. (1988), Vera y Cabanilla (1993), Martínez et al. (2000), es un factor muy importante para lograr una mayor polinización de flores, (Albores y Sosa, 2006).

No existió significancia estadística para la variable cosechados (Tabla 2); sin diferencias estadísticas ( $p>0,05)$ esto concuerda con lo descrito por Soria (1976) quien menciona que la presión de aire dirigido a los cojinetes florales ubicados en el tronco permitió liberar la mayor cantidad de polen seco, así también al aplicar la presión al suelo activó la actividad de mosquitas Forcypomnia ssp. en las huertas, conociendo que su hábitat natural son los cascarones de mazorcas sanos y pseudotallos de musáceas en estado de descomposición, por lo que estos insectos serían de alguna manera forzados a buscar los troncos, cojinetes y hojas (Páliz y Mendoza, 1993; León, 2000; Martínez , 2000) resultados similares lo obtuvo Guerrero et al. (2006) cuando seleccionaron manzanos con un $70 \%$ de germinación del polen. Lo que podría incrementar un mejor cuajado inicial de fruto para la cosecha comercial considerando también la disponibilidad y la densidad de floración (Lombard et al., 1988).

Para el peso total de fruto, se encontró significancia $(\mathrm{p} \geq 0,05)$ entre los T1, T2 y T3 $(922,96$, $938,09$ y $937,36 \mathrm{~g})$, respecto al T0 $(857,16)$ siendo el menor peso registrado, estos resultados del peso del fruto y las características cualitativas están relacionados con la forma, el diámetro ecuatorial y longitudinal, tamaño de la flor, y el incremento de la fecundación de las flores, permitiendo la obtención de nuevos frutos en el CCN-51 que se desarrollaran durante 5 a 6 meses en el tronco, y con una cosecha mayor de peso fresco (Vera, y Mogrovejo. 1979; Lombard et al., 1988; Klein et al., 2003).

\section{Número de semillas y rendimiento estimado $\mathrm{kg} \mathrm{ha}^{-1}$}

Para el número de semillas y rendimiento, no se encontró diferencias $(p \geq 0,05)$, por tratarse de la misma genética, resultados similares lo obtuvo Soria (1976), quien realizó estudios con cacao variedad Forastero en Brasil, logrando caracterizar la autocompatibilidad con la productividad, otros resultados parecidos se presentaron en México en cultivo de melón, determinando que la viabilidad del polen está relacionado con la calidad del fruto pues este refleja un mayor número de semillas (Reyes et al., 1982; Reyes et al., 2009).

Para el rendimiento los valores fueron cercanos, no se encontró diferencias estadísticas $(\mathrm{p} \geq 0,05)$, siendo para la presión de aire al suelo con $1.243,64 \mathrm{~kg} \mathrm{ha}^{-1}$, contrariamente el testigopolinización natural $1.227,44 \mathrm{~kg} \mathrm{ha}^{-1}$. Es posible que por tratarse de un mismo material genético en condiciones homogéneas, igual tamaño de flor (mediana y grande), reflejaría similar manera un aceptable tamaño de mazorca (Gingras et al., 1999), en días soleados con abundante floración, otros estudios en otras especias lo reportó Borba et al. (2002) quienes estudiaron la fecundación en 
orquídeas, logrando relacionar el tamaño de la flor con el tamaño final de la mazorca y su rendimiento final. No obstante Guerrero et al. (2006) realizó sus estudios con manzanos, expresando que una característica a considerar es la viabilidad del polen sobre un $70 \%$ de germinación. En consecuencia, la sincronía de floración incide directamente sobre el rendimiento y las condiciones medioambientales, esto coincide con la densidad de la floración, según Reyes et al. (2009).

\section{Conclusiones}

Se concluye que las polinizaciones artificiales, manual, presión de aire al tronco, obtuvieron mayor números de flores fecundadas existiendo significancia estadística, mientras para las demás variables número de semillas, rendimiento, frutos sanos, por tanto los métodos artificiales no influye sobre la producción final de la huerta de cacao clonal CCN-51 de siete años de edad.

\section{Literatura Citada}

Adu-Ampomah, Y.; Novak, F.; Afza, R.; Van Duren, M; PereaDallos, M. 1988. Initiation and Growth of Somatic Embryos of Cocoa (Theobroma cacao). The Café-Cacao, 32 (3): 187-199.

Albores, O. y Sosa, V.

2006. Polinización de dos especies simpátricas de steils (Pleurothallidinae, Orchidaceae). Acta Botánica Mexicana, 74: 155-168.

Borba, E.L.; Shepherd, G.J. y Van de Berg, C.

2002. Floral and vegetative morphometrics of five Pleurotallis (Orchidaceae) species: correlations with taxonomy, phylogeny, genetic variability and pollination systems. Ann. Bot., 90: 219-230.

Braudeau, J.

1970. El Cacao: Técnicas Agrícolas y Producciones Tropicales. Traducido del francés por Ángel Hernández Córdova. Primera edición. Blume. Barcelona, España, 297 p.

Crespo, C.E. y Crespo, A.F.

1997. Cultivo y Beneficios del Cacao CCN-51. Ed. Conejo Primera Edición. Quito, Ecuador, 136 p.

Enríquez, G.C.

2004. Cacao orgánico. Guía para productores ecuatorianos. La incompatibilidad en el cacao. Instituto Nacional de Investigaciones Agropecuarias. Manual No 54. Quito, Ecuador, $360 \mathrm{p}$.

Gingras, D.J.; Gingras, J. y Oliveira, D.

1999. Visits of honeybees (Hymenoptera: Apidae) and their effects on cucumber yield in the field. Hort. Entomol., 92 (2): 435-438

Guerrero, V.; Romo, A.; Orozco, J.A.; Berlanga, D.I.; Gardea,

A.; Parra, R.A.

2006. Polinización en manzanos "Red Delicius" y "Golden Delicius". Revista Fitotecnia. Mex,. 29 (1): 41-45.

Hardy, F.

1961. Botánica morfológica de la planta de cacao. En: Manual de cacao; Hardy, F. (Ed.). Instituto Interamericano de Ciencias Agrícolas de la OEA. Turrialba, Costa Rica, pp. 329-337.

Klein, A.M.; Streffan, I. y Tscharntke, T. 2003. Bee pollination an fruit set of Coffe Arabica and $C$. canephora (Rubiaceaea). Am. J. Bot., 90 (1): 153-157.

León, J.

2000. Botánica de los Cultivos Tropicales. Tercera edición. IICA. San José, Costa Rica, 678 p.
Lombard, P.B.; Callan, N.W.; Dennis Jr, F.G.; Looney, N.E.; Martin, G.C.; Renquist, A.R. y Mielke, E.A.

1988. Towards a standardized nomenclature, procedures, values, and units in determining fruit and tree yield performance. HortSience, 23 (5): 813-817.

Martínez, A.; Narváez, Z. y Spinelli, G. 2000. Mosquitas polinizadoras (Diptera: Ceratopogonidae) del cacao colectadas en comunidades piaroa en Amazonas, Venezuela. Bol Entomol Venez., 15 (2): 249-253.

Páliz, V. y Mendoza, J.

1993. Insectos del cacao. En: Manual del Cultivo del cacao. Manual No 25 Estación Experimental Tropical Pichilingue del INIAP Quito-Ecuador, pp. 109-116.

Quiroz, J. y Agama, J.

2005. Reproducción por medio de ramillas, Programa de capacitación de la cadena del cacao. Modulo: Producción del cacao. Ecuador, p. 28.

Quiroz, J.; Vera, J. y Enríquez, G.

1992. Determinación de genotipos de compatibilidad de algunos clones de cacao (Theobroma cacao L.). Boletín $N^{\circ}$ 71. Estación Experimental Tropical Pichilingue del INIAP. Quito, Ecuador, 10 p.

Reyes, J.L.; Cano J.L. y Nava, P.

2009. Período óptimo de polinización del melón con abejas melíferas (Apis melíferas L.) Agricultura Técnica en México, 35 (4): 370-377.

Reyes, J.L.; Valdéz, M.T. y Villa, D.M.

1982. La polinización por abejas (Apis mellifera L.) en el cultivo de melón (Cucumis melo L.) en la Lagunera. ALCA, 17 (1): 17-28.

SAS

2002. User's guide: Stadistic. Versión 9.0 Statical Analysis Sistem Institute. Cary, NC.

SICA (Servicio de Información y Censo Agropecuario)

2006. Importancia del cacao en Ecuador. Consultado el 29 de diciembre de 2011. Disponible en htt://www.sica.gov. ec.htm.

Soria, S. de J.

1976. Uso de atomizadores para inducir producão de cacaueiro. Revista Cacao Actualidades (12) 1: 18.

Soria, S. de J.

1977. Botánica morfológica de la planta de cacao. En: Manual de cacao, Hardy, F. (Ed). Instituto Interamericano de Ciencias Agrícolas de la OEA. Turrialba, Costa Rica, pp. 329-337. 
Urquhart, D.H.

1963. Cocoa Instituto Interamericano de Ciencias Agrícolas IICA. Turrialba, Costa Rica, 322 p.

Vera, J. y Mogrovejo, E.

1979. Aumente la producción de sus cacaotales haciendo polinización manual suplementaria. Estación Experimental
Tropical Pichilingue INIAP. Boletín Técnico No 36, Quito Ecuador, 8 p.

Vera, J. y Cabanilla, H.

1993. Rehabilitación de cacao. En: Manual del cultivo de cacao. Manual No 26 Estación Experimental Tropical Pichilingue del INIAP, Quito, Ecuador, pp. 118-124. 